\title{
An Empirical Study on the Use and Misuse of Java 8 Streams
}

\author{
Raffi Khatchadourian ${ }^{1,2}$ (D), Yiming Tang ${ }^{2}$, Mehdi Bagherzadeh ${ }^{3}$ (D), and \\ Baishakhi Ray ${ }^{4}$ (D) \\ ${ }^{1}$ CUNY Hunter College, New York, NY USA \\ 2 CUNY Graduate Center, New York, NY USA \\ $\{$ raffi.khatchadourian, ytang 3$\} @\{$ hunter, gradcenter $\}$. cuny.edu \\ 3 Oakland University, Rochester, MI USA \\ mbagherzadeh@oakland.edu \\ ${ }^{4}$ Columbia University, New York, NY USA \\ rayb@cs.columbia.edu
}

\begin{abstract}
Streaming APIs allow for big data processing of native data structures by providing MapReduce-like operations over these structures. However, unlike traditional big data systems, these data structures typically reside in shared memory accessed by multiple cores. Although popular, this emerging hybrid paradigm opens the door to possibly detrimental behavior, such as thread contention and bugs related to non-execution and non-determinism. This study explores the use and misuse of a popular streaming API, namely, Java 8 Streams. The focus is on how developers decide whether or not to run these operations sequentially or in parallel and bugs both specific and tangential to this paradigm. Our study involved analyzing 34 Java projects and 5.53 million lines of code, along with 719 manually examined code patches. Various automated, including interprocedural static analysis, and manual methodologies were employed. The results indicate that streams are pervasive, parallelization is not widely used, and performance is a crosscutting concern that accounted for the majority of fixes. We also present coincidences that both confirm and contradict the results of related studies. The study advances our understanding of streams, as well as benefits practitioners, programming language and API designers, tool developers, and educators alike.
\end{abstract}

Keywords: empirical studies $\cdot$ functional programming $\cdot$ Java $8 \cdot$ streams - multi-paradigm programming $\cdot$ static analysis.

\section{Introduction}

Streaming APIs are widely-available in today's mainstream Object-Oriented programming (MOOP) languages and platforms [5], including Scala [14], JavaScript [44], C\# [33], F\# [47], Java [39], and Android [27]. These APIs allow for "big data"-style processing of native data structures by incorporating MapReducelike [10] operations. A "sum of even squares" example in Java, where a stream of numbers is derived from a list, filtered for evens, mapped to its squared, and summed [5] is: list. $\operatorname{stream}() \cdot f i l t e r(x \rightarrow x \% 2==0) \cdot \operatorname{map}(x \rightarrow x * x) \cdot \operatorname{sum}()$.

(C) The Author(s) 2020

H. Wehrheim and J. Cabot (Eds.): FASE 2020, LNCS 12076, pp. 97-118, 2020.

https://doi.org/10.1007/978-3-030-45234-6_5 
Traditional big data systems, for which MapReduce is a popular backbone [3], minimize the complexity of writing massively distributed programs by facilitating processing on multiple nodes using succinct functional-like constructs. This makes writing parallel code easier, as writing such code can be difficult due to possible data races, thread interference, and contention [1,4,28]. The code above, e.g., can execute in parallel simply by replacing stream() with parallelstream().

However, unlike traditional big data systems, data structures processed by streaming APIs like Java 8 Streams typically reside in shared memory accessed by multiple cores. Therefore, issues may arise from the close intimacy between shared memory and the operations being performed, especially for developers not previously familiar with functional programming. Streams are not just an API but rather an emerging, hybrid paradigm. To obtain the expressiveness, speed, and parallelism that streams have to offer, developers must adopt the paradigm as well as the API [6, Ch. 7]. This requires determining whether running stream code in parallel yields an efficient yet interference-free program [24] and ensuring that no operations on different threads interleave [42].

Despite the benefits [53, Ch. 1], misusing streams may result in detrimental behavior, and the $\sim 4 \mathrm{~K}$ questions related to streams on Stack Overflow [48], of which $\sim 5 \%$ remain unanswered, suggest that there is ample confusion surrounding the topic. Bugs related to thread contention (due to $\lambda$-expressions, i.e., units of computation, side-effects, buffering), non-execution (due to deferred execution), non-determinism (due to non-deterministic operations), operation sequencing (ordering of stream operations), and data ordering (ordering of stream data) can lead to programs that undermine concurrency, underperform, are incorrect, and are inefficient. Worse yet, these problems may increase over time as streams rise in popularity, with Mazinanian et al. [32] finding a two-fold increasing trend in the adoption of $\lambda$-expressions, an essential part of streams.

This study explores the use and misuse of a popular and representative streaming API, namely, Java 8 Streams. We set out to understand the usage and bug patterns involving streams in real software. Particularly, we are interested in discovering (i) how developers decide whether to run streams sequentially or in parallel, (ii) common stream operations, (iii) common stream attributes and whether they are amenable to safe and efficient parallelization, (iv) bugs both specific and tangential to streams, (v) how often incorrect stream APIs were used, and (vi) how often stream APIs were misused and in which ways?

Knowing the kinds of bugs typically associated with streams can, e.g., help improve (automated) bug detection. Being aware of the typical usage patterns of streams can, e.g., improve code completion in IDEs. In general, the results (i) advance our understanding of this emerging hybrid paradigm, (ii) provide feedback to language and API designers for future API versions, (iii) help tool designers comprehend the struggles developers have with streams, (iv) propose preliminary best practices and anti-patterns for practitioners to use streaming APIs effectively, (v) and assist educators in teaching streaming APIs.

We analyzed 34 Java projects and 5.53 million lines of source code (SLOC), along with 140,446 code patches (git commits), of which 719 were manually 


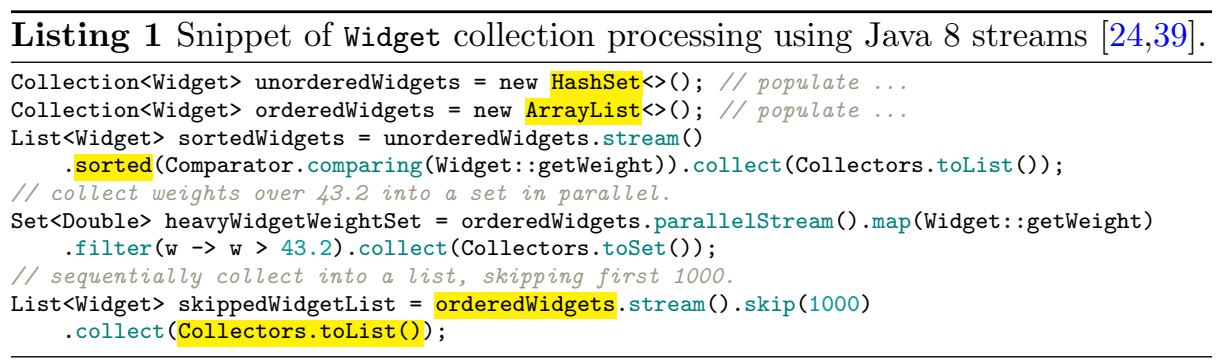

examined. The methodologies varied depending on the research questions and encompassed both automated, including interprocedural static analysis, and manual processes aided by automated software repository mining. Our study indicates that (i) streams have become widely used since their inception in 2014, (ii) developers tend to reduce streams back to iterative-style collections, favor simplistic, linear reductions, and prefer deterministic operations, (iii) stream parallelization is not widely used, yet streams tend not to have side-effects, (iv) performance is the largest category of stream bugs and is crosscutting.

This work makes the following contributions:

Stream usages patterns A large-scale analysis of stream and collector method calls and an interprocedural static analysis on 1.65 million lines source code is performed, reporting on attributes essential to efficient parallel execution.

Stream bug hierarchical taxonomy From the 719 git patches from 22 projects manually examined using 140 identifying keywords, we build a rich hierarchical, crosscutting taxonomy of common stream bugs and fixes.

Best practices and anti-patterns We propose preliminary best practices and anti-patterns of using streams in particular contexts from our statistical results as well as an in-depth analysis of first-hand conversations with developers.

\section{Motivating Example and Conceptual Background}

Lst. 1 portrays code that uses the Java 8 Stream API to process collections of Widgets (class not shown) with colors and weights. A Collection of Widgets is declared (line 1) that does not maintain element ordering as HashSet does not support it [38]. Note that ordering is dependent on the run time type.

A stream (a view representing element sequences supporting MapReduce-style operations) of unorderedWidgets is created on line 3. It is sequential, i.e., its operations will execute serially. Streams may also have an encounter order that may depend on its source. Here, it is unordered since HashSets are unordered.

On line 4, the stream is sorted by the corresponding intermediate operation, the result of which is a stream with the encounter order rearranged. Widget: : getWeight is a method reference denoting the comparison scheme. Intermediate operations are deferred until a terminal operation is executed like collect() (line 4). The collect() operation is a (mutable) reduction that aggregates results of prior intermediate operations into a given Collector. In this case, it is one that yields a List. The result is a Widget List sorted by weight. 
To potentially improve performance, this stream's "pipeline" (sequence of operations) may be executed in parallel. Note, however, that had the stream been ordered, running the pipeline in parallel may result in worse performance due to the multiple passes or data buffering required by stateful intermediate operations (SIOs) like sorted(). Because the stream is unordered, the reduction can be done more efficiently as the run time can use divide-and-conquer [39].

In contrast, line 2 instantiates an ArrayList, which maintains element ordering. Furthermore, a parallel stream is derived from this collection (line 6), with each Widget mapped to its weight, each weighted filtered (line 7), and the results collected into a Set. Unlike the previous example, however, no optimizations are available here as an SIO is not included in the pipeline and, as such, the parallel computation does not incur possible performance degradation.

Lines 9-10 create a list of Widgets gathered by (sequentially) skipping the first thousand from orderedWidgets. Like sorted(), skip() is also an SIO. Unlike the previous example, executing this pipeline in parallel could be counterproductive because the stream is ordered. It may be possible to unorder the stream (via unordered()) so that its pipeline would be more amenable to parallelization. In this situation, however, unordering could alter semantics as the data is assembled into a structure maintaining ordering. As such, the stream correctly executes sequentially as element ordering must be preserved.

This simplified example demonstrates that using streams effectively is not always straight-forward and can require complex (and interprocedural due to aliasing) analysis. It necessitates a thorough understanding of API intricacies, a problem that can be compounded in more extensive programs. As streaming APIs become more pervasive, it would be extremely valuable to MOOP developers not familiar with functional programming if statistical insight can be given on how best to use streams efficiently and how to avoid common bugs.

\section{Study Subjects}

At the core of our study is 34 open source Java projects that use streams. They vary widely in their domain and application, as well as size and popularity. All the subjects have their sources publicly available on GitHub and include popular libraries, frameworks, and applications. Many subjects were selected from previous studies $[20,21,22,24]$, others because they contained relatively diverse stream operations and exhibited non-trivial metrics, including stars, forks, and number of collaborators. It was necessary to use different subjects for different parts of the study due to the computationally intensive nature of some of the experiments. For such experiments, subjects were chosen so that the analysis could be completed in a reasonable time period with reasonable resources.

\section{Stream Characteristics}

We explore the typical usage patterns of streams, including the frequency of parallel vs. sequential streams and amenability to safe and efficient parallelism, by examining stream characteristics. This has important implications for understanding the use of this incredibly expressive and powerful language feature. It also offers insight into developers' perceived risks concerning parallel streams. 
Table 1. Stream characteristics.

\begin{tabular}{llllllllllll}
\hline subject & KLOC & age & eps & k str & seq & \multicolumn{2}{c}{ para ord } & unord se & SIO \\
\hline bootique & 4.91 & 4.18 & 362 & 4 & 14 & 14 & 0 & 11 & 3 & 4 & 0 \\
cryptomator & 7.99 & 6.05 & 148 & 3 & 12 & 12 & 0 & 11 & 1 & 2 & 0 \\
dari & 64.86 & 5.43 & 3 & 2 & 18 & 18 & 0 & 15 & 3 & 0 & 0 \\
elasticsearch & 585.71 & 10.03 & 78 & 6 & 210 & 210 & 0 & 165 & 45 & 10 & 0 \\
htm.java & 41.14 & 4.53 & 21 & 4 & 190 & 188 & 2 & 189 & 1 & 22 & 5 \\
JabRef & 138.83 & 16.36 & 3,064 & 2 & 301 & 290 & 11 & 239 & 62 & 9 & 0 \\
JacpFX & 23.79 & 4.71 & 195 & 4 & 12 & 12 & 0 & 9 & 3 & 1 & 0 \\
jdp & 19.96 & 5.53 & 25 & 4 & 38 & 38 & 0 & 35 & 3 & 11 & 1 \\
jdk8-exp & 3.43 & 6.35 & 34 & 4 & 49 & 49 & 0 & 47 & 2 & 5 & 0 \\
jetty & 354.48 & 10.93 & 106 & 4 & 57 & 57 & 0 & 47 & 10 & 8 & 0 \\
JetUML & 20.95 & 5.09 & 660 & 2 & 7 & 7 & 0 & 4 & 3 & 0 & 0 \\
jOOQ & 154.01 & 8.58 & 43 & 4 & 23 & 23 & 0 & 22 & 1 & 2 & 0 \\
koral & 7.13 & 3.47 & 51 & 3 & 8 & 8 & 0 & 8 & 0 & 0 & 0 \\
monads & 1.01 & 0.01 & 47 & 2 & 3 & 3 & 0 & 3 & 0 & 0 & 0 \\
retrolambda & 5.14 & 6.52 & 1 & 4 & 11 & 11 & 0 & 8 & 3 & 0 & 0 \\
spring & 188.46 & 11.62 & 5,981 & 4 & 61 & 61 & 0 & 60 & 1 & 21 & 0 \\
streamql & 4.01 & 0.01 & 92 & 2 & 22 & 22 & 0 & 22 & 0 & 2 & 18 \\
threeten & 27.53 & 7.01 & 36 & 2 & 2 & 2 & 0 & 2 & 0 & 0 & 0 \\
\hline Total & $1,653.35$ & 116.40 & 11,047 & 6 & 1,038 & 1,025 & 13 & 897 & 141 & 97 & 24 \\
\hline
\end{tabular}

${ }^{*}$ jdp is java-design-patterns, jdk8-exp is jdk8-experiments, spring is a portion of spring-framework, and threeten is threeten-extra.

\subsection{Methodology}

For this part of the study, we examined 18 projects that use streams, ${ }^{5}$ spanning $\sim 1.65$ million lines of Java source code. The subjects are depicted in tab. 1 . Column KLOC corresponds to thousands of source lines of code, which ranges from $\sim 1 \mathrm{~K}$ for monads to $\sim 586 \mathrm{~K}$ for elasticsearch. Column age is the age of the subject project in years, averaging 6.47 years per subject. Column str is the total number of streams analyzed. The remaining columns are discussed in $\S 4.2$.

Stream Pipeline Tracking Several factors contribute to determining stream attributes. First, streams are typically derived from a source (e.g., a collection) and take on its characteristics (e.g., ordering), as seen in lst. 1. There are several ways to create streams, including being derived from Collections, being created from arrays (e.g., Arrays.stream()), and via static factory methods (e.g., IntStream.range()). Second, stream attributes can change by the invocation of various intermediate operations in the building of the stream pipeline. Such attributes must be tracked, as it is possible to have arbitrary assignments of stream references to variables, as well as be data-dependent.

Our study involved tracking streams and their attributes (i.e., state) using a series of labeled transition systems (LTSs). The LTSs are fed into the static analysis portion of a refactoring tool [23] based on typestate analysis [16,49]. Stream pipelines are tracked and stream state when a terminal operation is issued is determined by the tool. Typestate analysis is a program analysis that augments the type system with "state" information and has been traditionally used for prevention of program errors such as those related to resource usage. It works by assigning each variable an initial $(\perp)$ state. Then, method calls transition the object's state. States are represented by a lattice and possible transitions

\footnotetext{
${ }^{5}$ Recall from $\S 3$ that it was necessary to use different subjects for different parts of
} the study due to the computationally intensive nature of some of the experiments. 
are represented by LTSs. If each method call sequence on the receiver does not eventually transition the object back to the $\perp$ state, the object may be left in a nonsensical state, indicating the potential presence of a bug.

The LTSs for execution mode and ordering work as follows. The state $\perp$ is a phantom initial state immediately before stream creation. Different stream creation methods may transition the newly created stream to one that is either sequential or parallel or ordered or unordered. The transition continues for each invoked intermediate operation and ends with a terminal operation.

Since the analysis is focused on client-side analysis of stream APIs, the call graph is constructed using a $k$-CFA, where $k$ is the call string length. It is an analysis parameter, with $k=2$ being the default, as it is the minimum $k$ needed to consider client-code, for methods returning streams and $k=1$ elsewhere. The refactoring tool includes heuristics for determining sufficient and tractable $k$.

Counting Streams Since stream attributes are control flow sensitive, the streams studied must be in the control flow of entry points. For non-library subjects, all main methods were chosen, otherwise, all unit tests were chosen.

Streams are counted as follows. First, every syntactic stream is counted, i.e., every allocation site. Streams in the control flow of the program starting from an entry point transition according to the LTSs. If a stream is not in the control flow, it is still counted but it remains at the state following $\perp$. This way, more information about various stream attributes is available for the study as we do not need control flow to determine the state following $\perp$.

Side-effects and Stateful Intermediate Operations Stream side-effects are determined using a ModRef analysis on stream operation parameters $(\lambda$ expressions) using WALA [52]. SIOs are obtained from the documentation [39].

\subsection{Results}

Tab. 1 illustrates our findings on stream characteristics. Column eps is the number of entry points. Column $\mathbf{k}$ is the maximum $k$ value used (see $\S 4.1$ ). Columns seq and para correspond to the number of sequential and parallel streams, respectively. Column ord is the number of streams that are ordered, i.e., those whose operations must maintain an encounter order, which can be detrimental to efficient parallel performance, while column unord is the number unordered streams. Column se is the number of stream pipelines that include sideeffects, which may induce race conditions. Finally, column SIO is the number of pipelines that include stateful intermediate operations, which may also be detrimental to efficient parallel performance.

\subsection{Discussion}

Parallel streams are not popular (1.25\%) despite their ease-of-use. Although Nielebock et al. [36] did not consider $\lambda$-expressions in stream contexts, this confirms that their findings extend into stream contexts. It may also coincide with the finding of Lu et al. [28], i.e., that developers tend to "think" sequentially.

Finding 1: Stream parallelization is not widely used.

When considering using parallel streams, it may also be important to consider the context. For example, many server applications deal with thread pools that 
span the JVM, and developers may be leery of the interactions of such pools with the underlying stream parallelization run time system. We found this to be the case with several pull requests $[15,45]$ that were issued by Khatchadourian et al. [24] as part of their refactoring evaluation to introduce parallel streams into existing projects. It may also be the case that the locations where streams operate are already fast enough or do not process significant amounts of data $[7,30]$. In fact, Naftalin [35, Ch. 6] found that there is a particular threshold in data size that must be reached to compensate for overhead incurred by parallel stream processing. Lastly, developers pointed us to several blog articles [54,59] expressing that parallel streams could be problematic under certain conditions.

There were, however, two projects that use parallel streams. Particularly, JabRef used the most parallel streams at 11. We conjecture that JabRef's use of parallel streams may stem from its status as a desktop application. Such applications typically are not managed by application containers and thus may not utilize global thread pools as in more traditional server applications.

Many streams are ordered $(86.42 \%)$, which can prevent optimal performance of parallel streams under certain conditions $[24,35,40]$. Thus, even if streams were run in parallel, they may not reap all of the benefits. This extends the findings of Nielebock et al. [36] that $\lambda$-expressions do not appear in contexts amenable to parallelization to streams for the case of ordering. Streams may still be amenable to parallelization, as $\S 5.2$ shows that many streams are traversed using API that ignores ordering (e.g., forEach() vs. forEach0rdered()).

Finding 2: Streams are largely ordered, possibly hindering parallelism.

That only $\sim 10 \%$ of streams have side-effects and only $2.31 \%$ have SIOs contradict the findings of Nielebock et al. [36] in the context of streams. This suggests that streams may run efficiently in parallel as, although they are largely ordered, they include minimal side-effects and SIOs. streamql had the most streams with SIOs (18/22), which may be due to its querying features using aggregate operations that are manifested as SIOs in the Java 8 Streaming API (e.g., distinct()).

Finding 3: Streams tend not to have side-effects.

\section{Stream Usage}

We discover the common operations on streams and the underlying reasons by examining stream method calls. This has important implications in understanding how streams are used, and studying language feature usage has been shown to be beneficial $[11,43]$. It provides valuable insight to programming language API designers and tool-support engineers on where to focus their evaluation efforts. We may also comprehend contexts where developers struggle with using streams.

\subsection{Methodology}

We examined 34 projects that use streams, spanning $\sim 5.53$ million lines of source code. To find method calls, we parsed ASTs with source-symbol bindings using the Eclipse Java Developer Tools (JDT) [12]. Then, method invocation nodes were extracted whose compile-time targets are declared in types residing in the java.util.stream package. This includes types such as Streams and Collectors. 
While stream creation is interesting and a topic for future work, our focus is on operations on streams as our scope is stream usage. We also combined methods with similar functionalities, e.g., mapToLong() with map() but not forEach() and forEachOrdered(). Additionally, only the method name is presented, resulting in a comparison of methods from both streams and collectors. The type is clear from the method name (e.g., map() is for Streams, while groupingBy() is for Collectors). We then proceeded to count the number of method calls in each project.

\subsection{Results}

Fig. 1 depicts the result of our analysis. ${ }^{6} \mathrm{~A}$ full table is available in our dataset [25]. The horizontal axis lists the method name, and the legend depicts projects analyzed. The chart is sorted by the total number of calls in descending order. Calls per project range from 4 for threeten-extra to 4,635 for cyclops. Calls per method range from 2 for characteristics(), which returns stream attributes such as whether it is ordered or parallel, and 3,161 for toList().

\subsection{Discussion}

The number of method calls in fig. 1 is substantial. There are 14,536 calls to methods operating on streams in 34 projects. This is impressive considering that Android, which uses the Java syntax, did not adopt streams immediately.

It is not surprising that the four most used stream methods are toList(), collect (), map(), and filter(), as these are the core MapReduce data transformation operations. $\operatorname{collect}()$ is a specialized reduction that reduces to a non-scalar type (e.g., a map) as opposed to the traditional scalar type. The toList() method is a static method of Collectors, which are pre-made reductions, in this case, to an ArrayList. This informs the collect() operation of the non-scalar type to use. It is peculiar that there are more calls to toList() than collect(). This is due to cyclops. We conjecture that it has some unorthodox usages of Collectors as it is a platform for writing functional-style programs in Java $\geq 8$ [2].

That collect() and toList(), along with other terminal operations such as forEach(), iterator(), toSet(), and toArray(), appear towards the top to the list suggest that, although developers are writing functional-style code to process data in a "big data" processing style, they are not staying there. Instead, they are "bridging" back to imperative-style code, either by collecting data into imperative-style collections or processing the data further iteratively.

There can be various reasons for this, such as unfamiliarity with functional programming, the need to introduce side-effects, or the need to interoperate with legacy code. Further investigation is necessary, yet, Nielebock et al. [36] mention that developers tend to introduce side-effects into $\lambda$-expressions, which is related.

Finding 4: Although stream usage is high, developers tend to reduce streams back to iterative-style collections.

We infer that developers tend to favor more simplistic (linear) rather than more specialized (higher-dimensionality) non-scalar reductions. It is surprising that more of the advanced reductions, such as those that return maps (e.g., toMap(),

${ }^{6}$ Similar conclusions hold when normalizing with subject KLOC. 


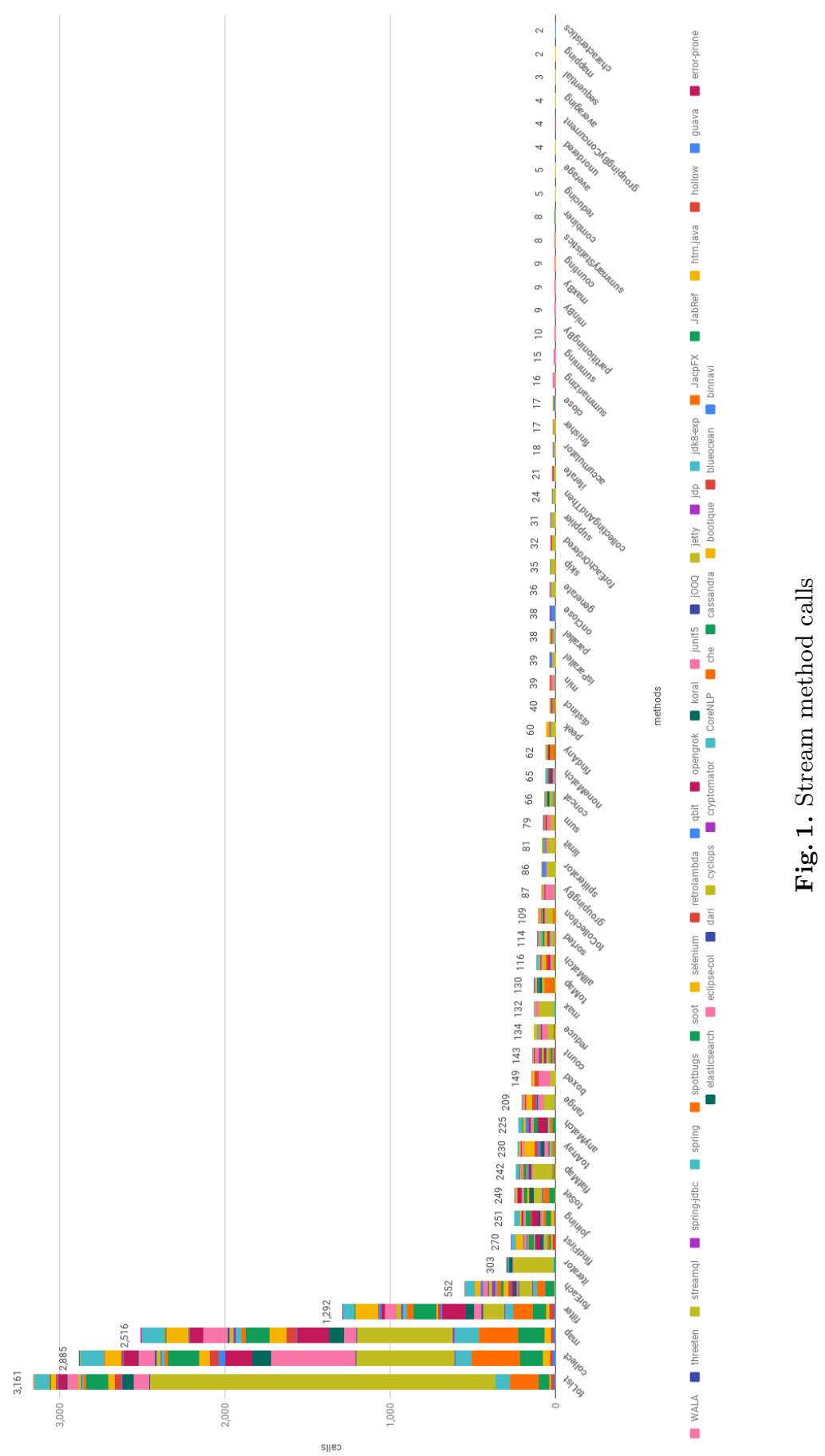


groupingBy()) are not used more frequently as these are highly expressive operations that can save substantial amounts of imperative-style code. For example, one may group Widgets by their Color as Map<Color, List<Widget >> widgetsByColor = widgets.stream().collect (Collectors.groupingBy(Widget: :getColor)). Although these advanced reductions are powerful and expressive, developers may be leery of using them, perhaps due to unfamiliarity or risk adversement. This motivates future tools that refactor to uses of advanced reductions to save developers time and effort while possibly mitigating errors.

Finding 5: Developers favor simplistic, linear reductions.

Another powerful stream feature is its non-determinism. For instance, findAny () returns any stream element. However, this operation has only 62 calls, while its deterministic counterpart, findFirst(), has 270, suggesting that developers tend to favor determinism. Yet, in contrast, developers overwhelmingly favor the nondeterministic forEach() operation (552) over the deterministic forEach0rdered() (32). We conjecture that although forEach() does not guarantee a particular ordering [41], in practice, since developers are inclined to use sequential over parallel streams, as suggested by $\S 4$ and mirrored by Nielebock et al. [36] in terms of $\lambda$-expressions, the difference does not play out.

It could also be that traversal order is largely unimportant for many streams. This is curious because, as demonstrated in $\S 4$, the majority of streams are ordered, an attribute detrimental to efficient parallelism $[24,35,40]$. As such, there may exist opportunities to alleviate the burden of stream ordering maintenance to make parallel streams more efficient. It may also entice developers to use more parallel streams as the performance gains may be significant.

Finding 6: Developers prefer deterministic operations.

Lastly, there is a minimal amount of calls to parallel stream APIs. Of particular concern is that there are only 4 calls to groupingByConcurrent() in contrast to the 87 calls to groupingBy(). This suggests that either advanced reductions to maps are not being used on parallel streams or that they are not used safely as the concurrent version provides synchronization [37]. Furthermore, not using groupingByConcurrent ( $)$ on a parallel stream may produce inefficient results [40].

\section{Stream Misuses}

This section is focused on discovering stream bug patterns. We are interested in bugs both specific and tangential to streams, i.e., bugs that occur in stream contexts. Understanding this can, e.g., help improve (automated) bug detection and other tool-support for writing optimal stream code. We may also begin to understand the kinds of errors developers make with streams, which may positively influence how future API and language feature versions are implemented.

\subsection{Methodology}

Here, we explore 22 projects that use streams, comprising $\sim 4.68$ million lines of source code and 140,446 git commits. ${ }^{7}$ Tab. 2 summarizes the subjects used.

\footnotetext{
${ }^{7}$ Recall from $\S 3$ that it was necessary to use different subjects for different parts of the study due to the computationally intensive nature of some of the experiments.
} 
Table 2. Studied subjects.

\begin{tabular}{lllrrr}
\hline subject & KLOC & studied periods & cmts & kws & exe \\
\hline binnavi & 328.28 & $2015-08-19$ to $2019-07-17$ & 286 & 4 & 4 \\
blueocean-plugin & 49.70 & $2016-01-23$ to $2019-07-24$ & 4,043 & 118 & 25 \\
bootique & 15.47 & $2015-12-10$ to $2019-08-08$ & 1,106 & 5 & 5 \\
che & 189.24 & $2016-02-11$ to $2019-08-19$ & 8,093 & 75 & 75 \\
cryptomator & 9.83 & $2014-02-01$ to $2019-08-08$ & 1,443 & 50 & 10 \\
dari & 72.46 & $2012-09-26$ to 2018-03-02 & 2,466 & 18 & 6 \\
eclipse.jdt.core & $1,527.89$ & $2001-06-05$ to 2019-08-07 & 24,085 & 234 & 106 \\
eclipse.jdt.ui & 712.91 & $2001-05-02$ to 2019-08-09 & 28,136 & 149 & 32 \\
error-prone & 165.85 & $2011-09-14$ to 2019-08-15 & 3,893 & 71 & 71 \\
guava & 393.47 & $2009-06-18$ to 2019-08-15 & 5,031 & 36 & 36 \\
htm.java & 41.63 & $2014-08-09$ to 2019-02-19 & 1,507 & 40 & 1 \\
JacpFX & 24.06 & $2013-08-12$ to 2018-04-27 & 365 & 37 & 14 \\
jdk8-experiments & 3.47 & $2013-08-03$ to 2018-03-10 & 8 & 1 & 1 \\
java-design-patterns & 33.52 & $2014-08-09$ to 2019-07-31 & 2,192 & 37 & 12 \\
jetty & 400.26 & $2009-03-16$ to 2019-08-02 & 17,051 & 835 & 219 \\
jOOQ & 184.25 & $2011-07-24$ to 2019-07-31 & 7,508 & 94 & 4 \\
qbit & 52.27 & $2014-08-25$ to 2018-01-18 & 1,717 & 65 & 9 \\
retrolambda & 5.10 & $2013-07-20$ to 2018-11-30 & 522 & 17 & 4 \\
selenium & 234.12 & $2004-11-03$ to 2019-08-09 & 24,145 & 114 & 57 \\
streamql & 4.26 & $2014-04-27$ to 2014-04-29 & 27 & 2 & 2 \\
threeten-extra & 31.26 & $2012-11-17$ to 2019-07-14 & 559 & 28 & 2 \\
WALA & 203.84 & $2006-11-22$ to 2019-07-24 & 6,263 & 52 & 24 \\
\hline Total & $4,683.12$ & & 140,446 & 2,082 & 719 \\
\hline
\end{tabular}

To find changesets (patches) corresponding to stream fixes, we compiled 140 keywords from the API documentation [39] that match stream operations and related method names from the java.util.stream package. We then randomly selected a subset of these commits whose changesets included these keywords and were likely to be bug fixes to manually examine.

Commit Mining To discover commits that had changesets including stream API keywords, we used gitcproc [9], a tool for processing and classifying git commits, which has been used in previous work $[17,50]$. Due to the keyword-based search used, not all of the examined commits pertained to streams (e.g., "map" has a broad range of applications outside of streams). To mitigate this, we focused more on keywords that were specific to stream contexts, e.g., "Collector." Also to reduce false positives, we only considered commits after the Java 8 release date of March 18, 2014, which is when streams were introduced.

Finding Bug Fixes We used a feature of gitcproc that uses heuristics based on commit log messages to identify commits that are bug fixes. Natural language processing (NLP) is used to determine which commits fall in this category. This helps us to focus on the likely bug-fix commits for further manual examination.

Next, the authors manually examine these commits to determine if the commits were indeed related to stream-related bugs. Three of the authors are software engineering and programming language professors with extensive expertise in streaming and parallel systems, concurrent systems, and empirical software engineering. The authors also have several years of industrial experience working as software engineers. As the authors did not always have expertise in the subject domains, only changes where a bug fix was extremely likely were marked as such. The authors also used commit comments and referenced bug databases to ascertain whether a change was a bug fix. This is a common practice $[8,26,28]$. 
Table 3. Stream bug/patch category legend.

\begin{tabular}{lll}
\hline name & description & acronym \\
\hline Bounds & Incorrect/Missing Bounds Check & BC \\
Exceptions & Incorrect/Missing Exception Handling & EH \\
Other & Other change (e.g., syntax, refactoring) & Other \\
Perf & Poor Performance & PP \\
Concur & Concurrency Issue & CI \\
Stream Source & Incorrect/Missing Stream Source & SS \\
Intermediate Operations Incorrect/Missing Intermediate Operations IO \\
Data Ordering & Incorrect Data Ordering & DO \\
Operation Sequencing & Incorrect Operation Sequencing & OS \\
Filter Operations & Incorrect/Missing Filter Operations & FO \\
Map Operations & Incorrect/Missing Map Operations & MO \\
Terminal Operations & Incorrect/Missing Terminal Operations & TO \\
Reduction Operations & Incorrect Reduction Operations & RO \\
Collector Operations & Incorrect/Missing Collector Operations & CO \\
Incorrect Action & Incorrect Action (e.g., $\lambda$-expression) & IA \\
\hline
\end{tabular}

Classifying Bug Fixes Once bug fixes were identified, the authors studied the code changes to determine the category of bug fixes and whether the category relates to streams. Fortunately, we found that many commits reference bug reports or provide more details about the fix. Such information proved highly valuable in understanding the fixes. When in doubt, we also sent emails to developers for clarification purposes as git commits include email addresses.

\subsection{Results}

Quantitative Column kws of tab. 2 is the number of commits where occurrences of keywords were found and correspond to possible stream bug fixes. Column exe depicts the number of commits manually examined. From these 719 commits, we found 61 stream client code bug fixes. This is depicted in column total of tab. 4 . Finding these bugs and understanding their relevance required a significant amount of manual labor that may not be feasible in more larger-scale, automated studies. Nevertheless, as streams become more popular (they were only introduced in 2014), we expect the usage and number of bugs related to streams to grow.

From the manual changes, we devised a set of common problem categories. Fixes were then grouped into these categories as shown in fig. 2 and tab. 4 . A category legend appears in tab. 3, where column name is the "short" name of the bug category and is used in fig. 2. Column description is the categories extended name and column acronym is used in tab. 4.

Fig. 2 presents a hierarchical categorization of the 61 stream-related bug fixes. Bugs are represented by their category name (column name in tab. 3) and their bug counts. Categories with no count are abstract, i.e., those grouping categories.

Bugs are separated into two top-level categories, namely, bugs specifically related to stream API usage (stream-specific) and those tangentially related, i.e., bugs appearing in stream contexts but not specifically having to do with streams (generic). Generic bugs were further categorized into related to exception handling $(\mathrm{EH})$, bounds checking $(\mathrm{BC})$, poor performance $(\mathrm{PP})$, and "other." Generic exception handling bugs (6) include those where, e.g., $\lambda$-expressions passed to stream operations threw exceptions that were not handled properly. Generic bounds checking bugs (2) included those where $\lambda$-expressions missed traversal boundary checks, and generic performance bugs (2) were those involving, 


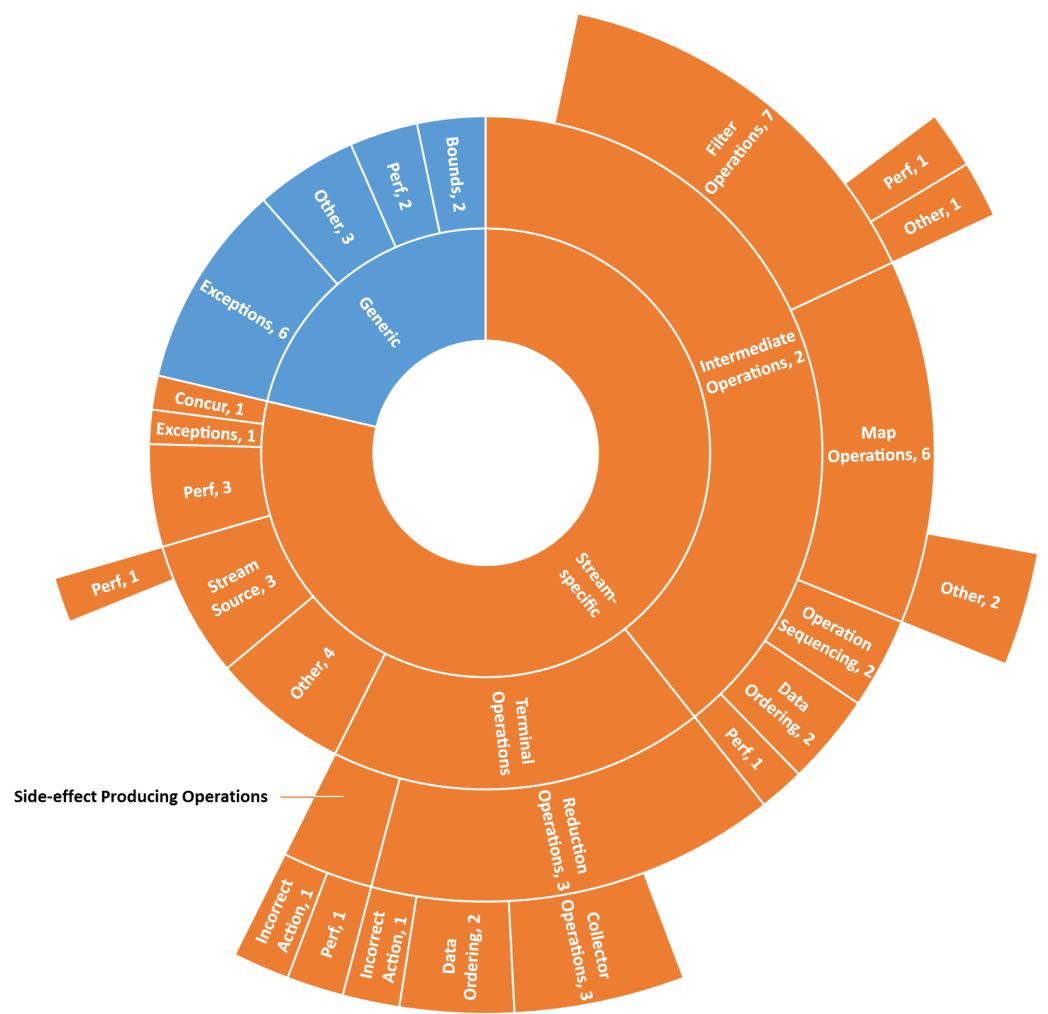

Fig. 2. Studied stream bugs and patches (hierarchical).

e.g., local variables holding stream computation results. The "other" category (3) is aligned with a similar one used by Tian and Ray [50] and involved syntactic corrections, e.g., incorrect types, and refactorings. Generally, "other" bugs can either be stream-specific or generic.

Stream-specific bugs are further divided into several categories corresponding to whether they involved intermediate operations (IO), terminal operations (TO), the stream source (SS), concurrency (CI), and performance and exception handling bugs specific to streams. IO-specific bugs (2) are related to intermediate operations other than filter operations (FO, 7) and map operations (MO, 6), e.g., distinct(). IO bugs are additionally partitioned into those involving incorrect operation sequencing (OS, 2), e.g., map() before filter(), data ordering (DO, 2), e.g., operating on a stream that should have been sorted, and performance bugs appearing in intermediate operations other than map() and filter() (1).

Terminal operations are split into two categories, namely, reduction operations (RO), e.g., collect(), reduce(), and side-effect producing operations, e.g., forEach(), iterator(). RO-specific bugs (3) were those related to scalar reductions, e.g., anyMatch(), allMatch(). RO-specific bugs related to collector operations $(\mathrm{CO}, 3)$, on the other hand, involve non-scalar reductions, e.g., a collector malfunction. RO-specific data ordering bugs (DO, 2) correspond to ordering of data related to scalar reductions, e.g., using findAny() instead of 
Table 4. Studied stream bugs and patches (nonhierarchical).

\begin{tabular}{|c|c|c|c|c|c|c|c|c|c|c|c|c|c|c|c|}
\hline subject & $\mathrm{BC}$ & $\mathrm{CI}$ & $\mathrm{CO}$ & $\mathrm{DO}$ & $\mathrm{EH}$ & $\mathrm{FO}$ & IA & $\mathrm{IO}$ & $\mathrm{MO}$ & OS & $\mathrm{PP}$ & $\mathrm{RO}$ & SS & Other & Total \\
\hline binnavi & & & & & & & & & & & & & & 1 & 1 \\
\hline blueocean-plugin & & & & & & & & & & & & & & 1 & 1 \\
\hline bootique & & & & & & & & & & & 1 & & & & 1 \\
\hline che & & & & & 1 & 1 & & & 1 & & & 1 & & & 4 \\
\hline cryptomator & 1 & & & & 2 & & & & & & 1 & & & 2 & 6 \\
\hline dari & & & & & & & & & & & & & & 2 & 2 \\
\hline eclipse.jdt.core & & & & & & & & & & & & & & 1 & 1 \\
\hline eclipse.jdt.ui & & & & & & & & & & & & & 1 & & 1 \\
\hline error-prone & & & & & 2 & 1 & 1 & & 3 & & 1 & 1 & 2 & 1 & 12 \\
\hline guava & & & & & & & & & & & & & & 1 & 1 \\
\hline JacpFX & & 1 & & 1 & & & & & & & 2 & & & & 4 \\
\hline jdp & & & & & & & & & & & 1 & & & & 1 \\
\hline jetty & & & 1 & 2 & & & & & 1 & & 3 & & & & 7 \\
\hline jOOQ & & & & & & & & & 1 & & & & & & 1 \\
\hline selenium & & & 2 & 1 & 2 & 5 & 1 & 2 & & 2 & & 1 & & 1 & 17 \\
\hline threeten-extra & 1 & & & & & & & & & & & & & & 1 \\
\hline Total & 2 & 1 & 3 & 4 & 7 & 7 & 2 & 2 & 6 & 2 & 9 & 3 & 3 & 10 & 61 \\
\hline
\end{tabular}

findFirst (). RO-specific incorrect actions (IA, 1) is where there is a problematic $\lambda$ in a scalar reduction, e.g., an incorrect predicate in noneMatch(). Side-effect producing operation bugs also include incorrect actions (IA, 1), e.g., a problematic $\lambda$ in forEach(). Such operations can also exhibit poor performance (PO, 1).

Some bug categories are crosscutting, appearing under multiple categories. An example is performance. For this reason, tab. 4 portrays a nonhierarchical view of fig. 2, which is also broken down by subject, including a column for each bug category regardless of its parent category (acronyms correspond to tab. 3).

Finding 7: Bugs, e.g., performance, crosscut concerns, affecting multiple categories, both specifically and tangentially, associated with streams.

Performance issues dominate the functional (excluding "other") bugs depicted in tab. 4, making up the categories "Performance/API misuse" and "Performance," accounting for $14.75 \%$ (9/61) of the bugs found. While some of these fixes were more cleaning-based (e.g., superfluous operations), others affected central parts of the system and were found during performance regression testing [56].

Finding 8: Although streams feature performance improving parallelism, developers tend to struggle with using streams efficiently.

Despite widespread performance issues, concurrency issues (CI), on the other hand, were not prevalent (1.64\%). The one concurrency bug was where a stream operation involved non-atomic variable access, which resulted in improper initialization [34]. Given that such a variable is accessed in a stream operation, however, it does indicate a possible side-effect and a need to consider refactoring such accesses to remove side-effects. This would make streams more amenable to efficient parallelization and perhaps promote more usage of parallel streams.

Finding 9: Concurrency issues were the least common streams bugs. However, concurrent variable access can cause thread contention, motivating future refactoring approaches that may promote more parallel streams.

The subjects selenium and error-prone had the most stream bugs with $27.87 \%$ and $19.67 \%$, respectively. We hypothesize that this is due to the relatively large size of these projects, as well as their high usage of streams. Specifically, they fell into the top ten in terms of KLOC and stream method calls in tab. 2 and fig. 1, 
respectively, with $\sim 400$ combined KLOC and 1,414 combined calls. Naturally, projects that use streams more are likely to have more bugs involving streams.

Qualitative We highlight several of the most common bug categories with examples, summarize common fixes, and propose preliminary best practices $(\mathbf{B P})$ and anti-patterns (AP). Due to space limitations, only a single example of each $\mathrm{BP} / \mathrm{AP}$ is shown; a complete set is available in our dataset [25]. Although some APs may seem applicable beyond streams, e.g., avoiding superfluous operations, we conjecture that streams are more prone to such patterns, e.g., due to the ease in which operations can be chained and the deferred execution they offer.

$S S \rightarrow P P$ Performance issues dominated the number of stream bugs found and also crosscut multiple categories. Consider the following performance regression [56]:

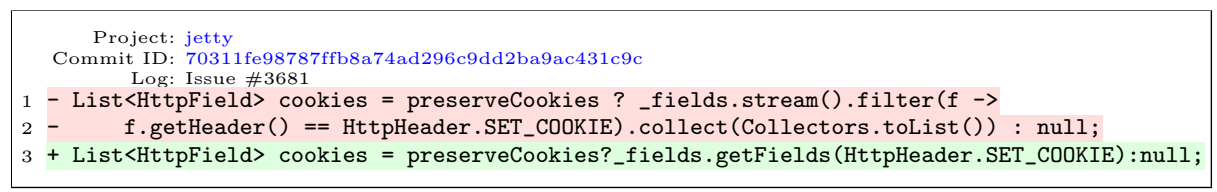

The stream field is replaced with getFields(), which performs an iterative traversal, effectively replacing streams with iteration. The developer found that using iteration was faster than using streams [57] and wanted more "JIT-friendly" code. The developer further admitted that using streams can make code more easy to read but can also be associated with "allocation/complexity cost [55]."

BP1: Use performance regression testing to verify that streams in critical code paths perform efficiently.

In the following, a pair of superfluous operations are removed:

Project: JacpFX

Commit ID: 4f0d62d3a0987e47a4cbdf8e056bdf89713e6aac

Log: fixed class scanning

final Stream<String > componentIds = CommonUtil

-getStringStreamFromArray (annotation.perspectives ());

final Stream<Injectable> perspectiveHandlerList =

componentIds parallel() . sequential () . map (this : :mapToInjectable) ;

componentIds.map (this : :mapToInjectable);

getStringStreaFromArray() returns a sequential stream, which is then converted to parallel and then to sequential. The superfluous operations are then removed.

$\boldsymbol{A P 1}$ : Avoid superfluous intermediate operations.

Fix: Generally, fixes for performance problems varied widely. They ranged from replacing stream code with iterative code, as seen above, to removing operations, to changing the stream source representations. Depending on context, the bugs' effect can be either innocuous and cause server performance degradation.

$S S \rightarrow T O \rightarrow R O \rightarrow C O$ The stream API provides several ready-made collectors for convenience. However, the API does not guarantee a specific non-scalar used during the reduction. On one hand, this is convenient as developers may not need a specific collection type; on the other hand, however, developers must be careful to ensure that the specific subclass returned by the API meets their needs.

In the following, the developer does not realize, until an incorrect program output, that the Map returned by Collectors.toMap() does not support nulls: 


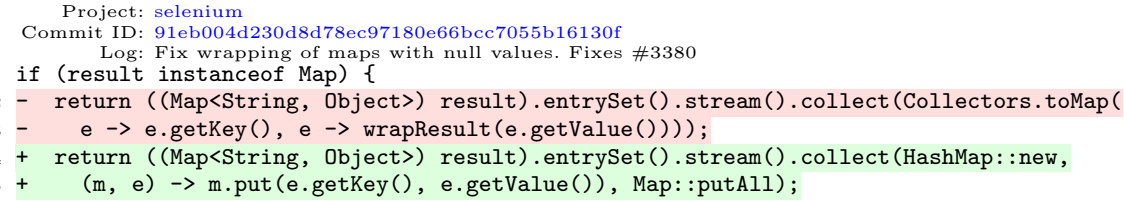

The ready-made collector (line 2) is replaced with a direct call to collect() with a particular Map implementation specified (line 4), i.e., HashMap.

BP2: Use collectors only if client code is agnostic to particular container implementations. Otherwise, use the direct form of collect().

Fix: Collector-related bugs are typically corrected by not using a Collector (as above), changing the Collector used, or altering the Collector arguments. They often adversely affect program behavior but are also caught by unit tests.

$S S \rightarrow I O$ In the ensuing commit, distinct() is called on a concatenated stream to ensure that no duplicates are created as a result of the concatenation:

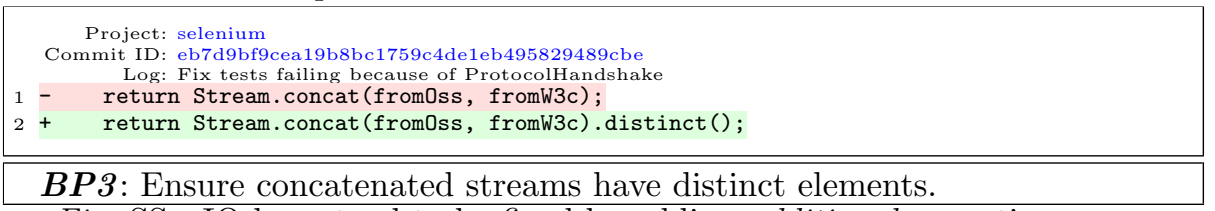

Fix: $\mathrm{SS} \rightarrow \mathrm{IO}$ bugs tend to be fixed by adding additional operations.

$S S \rightarrow I O \rightarrow$ Other Developers "bridged" back to an imperative-style performed an operation, then switched back to streams to continue a more functional-style:

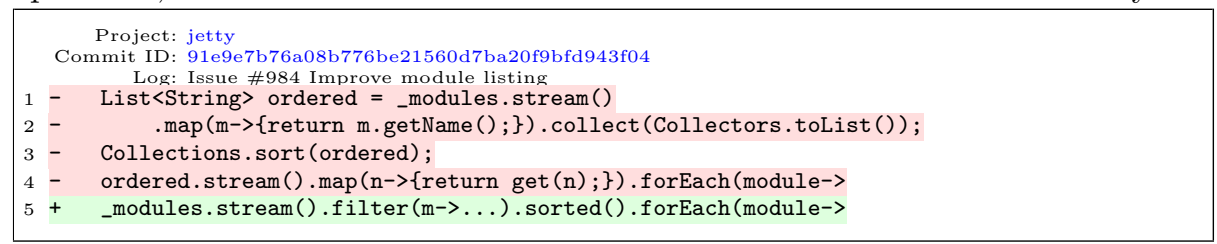

Each module is mapped to its name and collected into a list. Then, ordered is sorted via a non-stream Collections API. Another stream is then derived from ordered to perform further operations. However, on line 5 , the bridge to a collection and subsequent sort operation is removed, and the computation remains within the stream API. It is now more amenable to parallelization.

AP2: Avoid "bridging" between stream API and legacy collection APIs.

Using a long $\lambda$-expression in a single map() operation may make stream code less "functional," more difficult to read [29], and less amenable to parallelism. Consider the abbreviated commit below that returns the occupied drive letters on Windows systems by collecting the first uppercase character of the path:

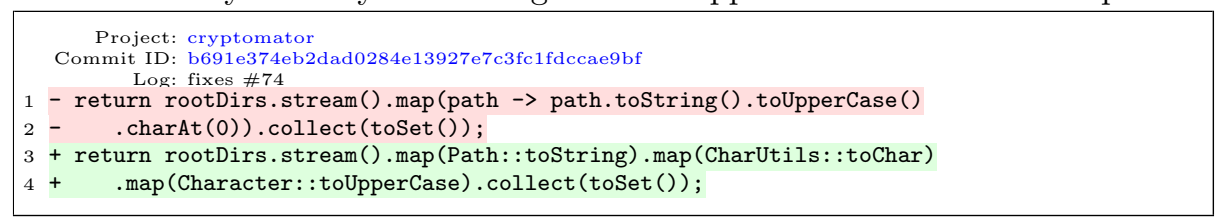

The $\lambda$-expression has been replaced with method references, however, there are more subtle yet import changes. Firstly, as CharUtils.toChar() returns the 
first character of a String, there is a small performance improvement as the entire string is no longer turned to uppercase but rather only the first character. Also, the new version is written in more of a functional-style by replacing the single $\lambda$-expression passed to map() with multiple map () operations. How data is transformed in the pipeline is easily visible, and future data transformations can be easily integrated by simply adding operations.

AP3: Avoid too many operations within a single map() operation.

Fix: "Other" non-type correcting fixes, e.g., refactorings, included introducing streams, sometimes from formerly iterative code (3), replacing map() with mapToInt () [20], and dividing "larger" operations into smaller ones.

\section{Threats to Validity}

Subjects may not be representative. To mitigate this, subjects were chosen from diverse domains and sizes. They have also been used in previous studies (e.g., [20,22]). Although java-design-patterns is artificial, it is a reference implementation similar to that of JHotDraw, which has been studied extensively (e.g., [31]). Also, as streams are relatively new, we expect a larger selection of subjects as they grow in popularity.

Entry points may not be correct, which could affect how stream attributes are calculated. Since standard entry points were chosen, these represent a superset of practically true entry points. Furthermore, there may be custom streams or collectors outside the standard API that we are not considered. As we aim to understand stream usage and misuse in the large, we hypothesize that the vast majority of projects using streams use ones from the standard libraries.

Our study involved many hours of manual validation, which can be subject to bias. However, we investigated referenced bug reports and other comments from developers to help us understand changes more fully. We also reached out to several developers via email correspondence when in doubt. All but one returned the correspondence. The NLP features of gitcproc may have missed changesets that were indeed bug fixes. Nevertheless, we were still able to find 61 bugs that contributed to a rich bug categorization, best practices, and anti-patterns. Furthermore, gitcproc has been used previously in other studies.

\section{Related Work}

Previous studies $[29,32,36,46,51]$ have focused specifically on $\lambda$-expressions. While $\lambda$-expressions are used as arguments to stream operations, our focus is on stream operations themselves. Such operations transition streams to different states, which can be detrimental to parallel performance [24,35]. Also, since streams can be aliased, we use a tool [24] based on typestate analysis to obtain stream attributes more reliably than AST-based approaches. We also study bugs related to stream usage and present developer feedback - fixing bugs related to streams may not involve changing $\lambda$-expressions; bugs can be caused by, e.g., an incorrect sequence of stream operations. Lastly, although Nielebock et al. [36] consider $\lambda$-expressions in "concurrency contexts," such contexts do not include streams, where $\lambda$-expressions can easily execute in parallel with minimal syntactical effort. 
Khatchadourian et al. [24] report on some stream characteristics as part of their refactoring evaluation but do so on a much smaller-scale, as their focus was on the refactoring algorithm. The work presented here goes significantly above in beyond by reporting on a richer set of stream characteristics (e.g., execution mode, ordering), with a noteworthy larger and updated corpus. We also include a comprehensive categorization of stream-related bug fixes, with 719 commits manually analyzed. Preliminary best practices and anti-patterns are also proposed.

Zhou et al. [60] conduct an empirical study on 210 service quality issues of a big data platform at Microsoft to understand their common symptoms, causes, and mitigations. They identify hardware faults, systems, and customer side effects as major causes of quality issues. There are also empirical studies on data-parallel programs. Kavulya et al. [19] study failures in MapReduce programs. Jin et al. [18] study performance slowdowns caused by system side inefficiencies. Xiao et al. [58] conduct a study on commutativity, nondeterminism, and correctness of data-parallel programs, revealing that non-commutative reductions lead to bugs. Though related, our work specifically focuses on stream APIs as a language feature and programming paradigm, which pose special considerations due to its shared memory model, i.e., interactions between the operations and local memory. Bloch [6, Ch. 7] also puts-forth stream best practices and anti-patterns. However, ours are based on a statistical analysis of real-world software and first-hand interactions with real-world developers.

Others also study language features. Parnin et al. [43] study the adoption of Java generics. Dyer et al. [11] build an expansive infrastructure for studying the use of language features over time. Khatchadourian and Masuhara [22] employ a proactive approach in empirically assessing new language features and present a case study on default methods. There are also many studies regarding bug analysis. For example, Engler et al. [13] present a general approach to inferring errors in systems code, and Tian and Ray [50] study error handling bugs in C.

\section{Conclusion \& Future Work}

This study advances our understanding of stream usage and bug patterns. We have surveyed common stream operations, attributes, and bugs specific and tangentially related to streams. A hierarchical taxonomy of stream bugs was devised, preliminary best practices and anti-patterns were proposed, and firsthand developer interactions were detailed. In the future, we will explore stream creation, use our findings to devise automated error checkers, and explore topics that interest stream developers. Lastly, we will investigate applicability to other streaming frameworks and languages.

Acknowledgments We would like to thank Krishna Desai and Robert Dyer for work on data summarization and discussions, respectively. Support for this project was provided by PSC-CUNY Award \#617930049, jointly funded by The Professional Staff Congress and The City University of New York. This material is based upon work supported by the National Science Foundation under Grant No. CCF 1845893, CNS 1842456, and CCF 1822965. 


\section{References}

1. Ahmed, S., and Bagherzadeh, M.: What Do Concurrency Developers Ask About?: A Large-scale Study Using Stack Overflow. In: International Symposium on Empirical Software Engineering and Measurement, 30:1-30:10 (2018). DOI: 10.1145/3239235. 3239524

2. AOL: AOL/cyclops: An advanced, but easy to use, platform for writing functional applications in Java 8. (2019). http://git.io/fjxzF (visited on 08/29/2019)

3. Bagherzadeh, M., and Khatchadourian, R.: Going Big: A Large-scale Study on What Big Data Developers Ask. In: Joint Meeting on European Software Engineering Conference and Symposium on the Foundations of Software Engineering. ESEC/FSE 2019, pp. 432-442. ACM, Tallinn, Estonia (2019). DOI: 10.1145/3338906.3338939

4. Bagherzadeh, M., and Rajan, H.: Order Types: Static Reasoning About Message Races in Asynchronous Message Passing Concurrency. In: International Workshop on Programming Based on Actors, Agents, and Decentralized Control, pp. 21-30 (2017). DOI: $10.1145 / 3141834.3141837$

5. Biboudis, A., Palladinos, N., Fourtounis, G., and Smaragdakis, Y.: Streams a la carte: Extensible Pipelines with Object Algebras. In: European Conference on ObjectOriented Programming, pp. 591-613 (2015). DOI: 10.4230/LIPIcs.ECOOP.2015.591

6. Bloch, J.: Effective Java. Prentice Hall, Upper Saddle River, NJ, USA (2018)

7. Bordet, S.: Pull Request \#2837 • eclipse/jetty.project, Webtide. (2018). http: //git.io/JeBAF (visited on 10/20/2019)

8. Casalnuovo, C., Devanbu, P., Oliveira, A., Filkov, V., and Ray, B.: Assert Use in GitHub Projects. In: International Conference on Software Engineering. ICSE '15, pp. 755-766. IEEE Press, Florence, Italy (2015). http://dl.acm.org/citation.cfm? $\mathrm{id}=2818754.2818846$

9. Casalnuovo, C., Suchak, Y., Ray, B., and Rubio-González, C.: GitcProc: A Tool for Processing and Classifying GitHub Commits. In: International Symposium on Software Testing and Analysis. ISSTA 2017, pp. 396-399. ACM, Santa Barbara, CA, USA (2017). DOI: 10.1145/3092703.3098230

10. Dean, J., and Ghemawat, S.: MapReduce: Simplified Data Processing on Large Clusters. Commun. ACM 51(1), 107-113 (2008). DOI: 10.1145/1327452.1327492

11. Dyer, R., Rajan, H., Nguyen, H.A., and Nguyen, T.N.: Mining Billions of AST Nodes to Study Actual and Potential Usage of Java Language Features. In: International Conference on Software Engineering. ICSE 2014, pp. 779-790. ACM, Hyderabad, India (2014)

12. Eclipse Foundation: Eclipse Java development tools (JDT), Eclipse Foundation. (2019). http://eclipse.org/jdt (visited on 10/19/2019)

13. Engler, D., Chen, D.Y., Hallem, S., Chou, A., and Chelf, B.: Bugs As Deviant Behavior: A General Approach to Inferring Errors in Systems Code. In: Symposium on Operating Systems Principles. SOSP '01, pp. 57-72. ACM, Banff, Alberta, Canada (2001). DOI: 10.1145/502034.502041

14. EPFL: Collections-Mutable and Immutable Collections-Scala Documentation, (2017). http://scala-lang.org/api/2.12.3/scala/collection/index.html (visited on 08/24/2018)

15. Erdfelt, J.: Pull Request \#2837 • eclipse/jetty.project, Eclipse Foundation. (2018). http://git.io/JeBAM (visited on 10/20/2019)

16. Fink, S.J., Yahav, E., Dor, N., Ramalingam, G., and Geay, E.: Effective Typestate Verification in the Presence of Aliasing. ACM Transactions on Software Engineering and Methodology 17(2), 91-934 (2008). DOI: 10.1145/1348250.1348255 
17. Gharbi, S., Mkaouer, M.W., Jenhani, I., and Messaoud, M.B.: On the Classification of Software Change Messages Using Multi-label Active Learning. In: Symposium on Applied Computing. SAC '19, pp. 1760-1767. ACM, Limassol, Cyprus (2019). DOI: $10.1145 / 3297280.3297452$

18. Jin, H., Qiao, K., Sun, X.-H., and Li, Y.: Performance Under Failures of MapReduce Applications. In: International Symposium on Cluster, Cloud and Grid Computing. CCGRID '11, pp. 608-609. IEEE Computer Society, Washington, DC, USA (2011). DOI: $10.1109 /$ ccgrid.2011.84

19. Kavulya, S., Tan, J., Gandhi, R., and Narasimhan, P.: An Analysis of Traces from a Production MapReduce Cluster. In: International Conference on Cluster, Cloud and Grid Computing. CCGrid 2010, pp. 94-103. IEEE, Melbourne, Australia (2010). DOI: 10.1109/CCGRID.2010.112

20. Ketkar, A., Mesbah, A., Mazinanian, D., Dig, D., and Aftandilian, E.: Type Migration in Ultra-large-scale Codebases. In: International Conference on Software Engineering. ICSE '19, pp. 1142-1153. IEEE Press, Montreal, Quebec, Canada (2019). DOI: 10.1109/ICSE.2019.00117

21. Khatchadourian, R., and Masuhara, H.: Automated Refactoring of Legacy Java Software to Default Methods. In: International Conference on Software Engineering, pp. 82-93 (2017). DOI: 10.1109/ICSE.2017.16

22. Khatchadourian, R., and Masuhara, H.: Proactive Empirical Assessment of New Language Feature Adoption via Automated Refactoring: The Case of Java 8 Default Methods. In: International Conference on the Art, Science, and Engineering of Programming, 6:1-6:30 (2018). DOI: 10.22152/programming-journal.org/2018/2/6

23. Khatchadourian, R., Tang, Y., Bagherzadeh, M., and Ahmed, S.: A Tool for Optimizing Java 8 Stream Software via Automated Refactoring. In: International Working Conference on Source Code Analysis and Manipulation, pp. 34-39 (2018). DOI: 10.1109/SCAM.2018.00011

24. Khatchadourian, R., Tang, Y., Bagherzadeh, M., and Ahmed, S.: Safe Automated Refactoring for Intelligent Parallelization of Java 8 Streams. In: International Conference on Software Engineering. ICSE '19, pp. 619-630. IEEE Press (2019). DOI: 10.1109/ICSE.2019.00072

25. Khatchadourian, R., Tang, Y., Bagherzadeh, M., and Ray, B.: An Empirical Study on the Use and Misuse of Java 8 Streams, (2020). DOI: 10.5281/zenodo.3677449. Feb. 2020.

26. Kochhar, P.S., and Lo, D.: Revisiting Assert Use in GitHub Projects. In: International Conference on Evaluation and Assessment in Software Engineering. EASE'17, pp. 298-307. ACM, Karlskrona, Sweden (2017). DOI: 10.1145/3084226.3084259

27. Lau, J.: Future of Java 8 Language Feature Support on Android. Android Developers Blog (2017). http://android-developers.googleblog.com/2017/03/future-of-java-8language-feature.html (visited on 08/24/2018)

28. Lu, S., Park, S., Seo, E., and Zhou, Y.: Learning from Mistakes: A Comprehensive Study on Real World Concurrency Bug Characteristics. In: International Conference on Architectural Support for Programming Languages and Operating Systems, pp. 329-339. ACM (2008). DOI: 10.1145/1346281.1346323

29. Lucas, W., Bonifácio, R., Canedo, E.D., Marcílio, D., and Lima, F.: Does the Introduction of Lambda Expressions Improve the Comprehension of Java Programs? In: Brazilian Symposium on Software Engineering. SBES 2019, pp. 187-196. ACM, Salvador, Brazil (2019). DOI: 10.1145/3350768.3350791

30. Luontola, E.: Pull Request \#140 • orfjackal/retrolambda, Nitor Creations. (2018). http://git.io/JeBAQ (visited on 10/20/2019) 
31. Marin, M., Moonen, L., and Deursen, A. van: An Integrated Crosscutting Concern Migration Strategy and its Application to JHotDraw. In: International Working Conference on Source Code Analysis and Manipulation (2007)

32. Mazinanian, D., Ketkar, A., Tsantalis, N., and Dig, D.: Understanding the Use of Lambda Expressions in Java. Proc. ACM Program. Lang. 1(OOPSLA), 85:1-85:31 (2017). DOI: $10.1145 / 3133909$

33. Microsoft: LINQ: .NET Language Integrated Query, (2018). http://msdn.microsoft. com/en-us/library/bb308959.aspx (visited on 08/24/2018)

34. Moncsek, A.: allow OnShow when Perspective is initialized, fixed issues with OnShow/OnHide in perspective • JacpFX/JacpFX@f2d92f7, JacpFX. (2015). http://git.io/Je0X8 (visited on 10/24/2019)

35. Naftalin, M.: Mastering Lambdas: Java Programming in a Multicore World. McGrawHill (2014)

36. Nielebock, S., Heumüller, R., and Ortmeier, F.: Programmers Do Not Favor Lambda Expressions for Concurrent Object-oriented Code. Empirical Softw. Engg. 24(1), 103-138 (2019). DOI: 10.1007/s10664-018-9622-9

37. Oracle: Collectors (Java Platform SE 10 \& JDK 10)-groupingByConcurrent, (2018). http://docs.oracle.com/javase/10/docs/api/java/util/stream/Collectors.html\# groupingByConcurrent(java.util.function.Function) (visited on 08/29/2019)

38. Oracle: HashSet (Java SE 9) \& JDK 9, (2017). http://docs.oracle.com/javase/9/ docs/api/java/util/HashSet.html (visited on 04/07/2018)

39. Oracle: java.util.stream (Java SE 9 \& JDK 9), (2017). http://docs.oracle.com/ javase/9/docs/api/java/util/stream/package-summary.html (visited on 02/22/2020)

40. Oracle: java.util.stream (Java SE 9 \& JDK 9)-Parallelism, (2017). http://docs.oracle. com/javase/9/docs/api/java/util/stream/package-summary.html\#Parallelism (visited on $02 / 22 / 2020$ )

41. Oracle: Stream (Java Platform SE 10 \& JDK 10)-forEach, (2018). http://docs. oracle.com/javase/10/docs/api/java/util/stream/Stream.html\#forEach(java.util. function.Consumer) (visited on 08/29/2019)

42. Oracle: Thread Interference, (2017). http://docs.oracle.com/javase/tutorial/ essential/concurrency/interfere.html (visited on 04/16/2018)

43. Parnin, C., Bird, C., and Murphy-Hill, E.: Adoption and Use of Java Generics. Empirical Softw. Engg. 18(6), 1047-1089 (2013). DOI: 10.1007/s10664-012-9236-6

44. Refsnes Data: JavaScript Array map() Method, (2015). http://w3schools.com/ jsref/jsref_map.asp (visited on 02/22/2020)

45. Rutledge, P.: Pull Request \#1 • RutledgePaulV/monads, Vodori. (2018). http: //git.io/JeBAZ (visited on 10/20/2019)

46. Sangle, S., and Muvva, S.: On the Use of Lambda Expressions in 760 Open Source Python Projects. In: Joint Meeting on European Software Engineering Conference and Symposium on the Foundations of Software Engineering. ESEC/FSE 2019, pp. 1232-1234. ACM, Tallinn, Estonia (2019). DOI: 10.1145/3338906.3342499

47. Shilkov, M.: Introducing Stream Processing in F\#, (2016). http://mikhail.io/2016/ 11/introducing-stream-processing-in-fsharp (visited on 07/18/2018)

48. Stack Overflow: Newest 'java-stream' Questions, (2018). http://stackoverflow.com/ questions/tagged/java-stream (visited on 03/06/2018)

49. Strom, R.E., and Yemini, S.: Typestate: A programming language concept for enhancing software reliability. IEEE Transactions on Software Engineering SE12(1), 157-171 (1986). DOI: 10.1109/tse.1986.6312929

50. Tian, Y., and Ray, B.: Automatically Diagnosing and Repairing Error Handling Bugs in C. In: Joint Meeting on European Software Engineering Conference and 
Symposium on the Foundations of Software Engineering. ESEC/FSE 2017, pp. 752762. ACM, Paderborn, Germany (2017). DOI: 10.1145/3106237.3106300

51. Uesbeck, P.M., Stefik, A., Hanenberg, S., Pedersen, J., and Daleiden, P.: An empirical study on the impact of $\mathrm{C}++$ lambdas and programmer experience. In: International Conference on Software Engineering. ICSE '16, pp. 760-771. ACM, Austin, Texas (2016). DOI: 10.1145/2884781.2884849

52. WALA Team: T.J. Watson Libraries for Analysis, (2015). http://wala.sf.net (visited on $01 / 18 / 2017$ )

53. Warburton, R.: Java 8 Lambdas: Pragmatic Functional Programming (2014)

54. Weiss, T.: Java 8: Behind The Glitz and Glamour of The New Parallelism APIs. OverOps Blog (2014). http://blog.overops.com/new-parallelism-apis-in-java- 8behind-the-glitz-and-glamour (visited on 10/20/2019)

55. Wilkins, G.: Issue \#3681 • eclipse/jetty.project@70311fe, Webtide, LLC. (2019)

56. Wilkins, G.: Jetty 9.4.x 3681 http fields optimize by gregw • Pull Request \#3682 • eclipse/jetty.project, Webtide, LLC. (2019). http://git.io/JeBAq (visited on $09 / 18 / 2019$ )

57. Wilkins, G.: Jetty 9.4.x 3681 http fields optimize by gregw • Pull Request \#3682 • eclipse/jetty.project. Comment, Webtide, LLC. (2019). http://git.io/ Je0MS (visited on 10/24/2019)

58. Xiao, T., Zhang, J., Zhou, H., Guo, Z., McDirmid, S., Lin, W., Chen, W., and Zhou, L.: Nondeterminism in MapReduce Considered Harmful? An Empirical Study on Non-commutative Aggregators in MapReduce Programs. In: ICSE Companion, pp. $44-53$ (2014). DOI: $10.1145 / 2591062.2591177$

59. Zhitnitsky, A.: How Java 8 Lambdas and Streams Can Make Your Code 5 Times Slower. OverOps Blog (2015). http://blog.overops.com/benchmark-how-java- 8lambdas-and-streams-can-make-your-code-5-times-slower (visited on 10/20/2019)

60. Zhou, H., Lou, J.-G., Zhang, H., Lin, H., Lin, H., and Qin, T.: An Empirical Study on Quality Issues of Production Big Data Platform. In: International Conference on Software Engineering. ICSE 2015, pp. 17-26. ACM, Florence, Italy (2015)

Open Access This chapter is licensed under the terms of the Creative Commons Attribution 4.0 International License (http://creativecommons.org/licenses/by/4.0/), which permits use, sharing, adaptation, distribution and reproduction in any medium or format, as long as you give appropriate credit to the original author(s) and the source, provide a link to the Creative Commons license and indicate if changes were made.

The images or other third party material in this chapter are included in the chapter's Creative Commons license, unless indicated otherwise in a credit line to the material. If material is not included in the chapter's Creative Commons license and your intended use is not permitted by statutory regulation or exceeds the permitted use, you will need to obtain permission directly from the copyright holder.

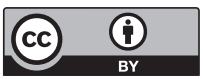

